\title{
KHK-Risiko bei Diabetikern: Der Blutdruck entscheidet
}

\begin{abstract}
Man geht davon aus, dass $40-80 \%$ der Diabetiker auch einen Bluthochdruck haben und damit deutlich häufiger als die Allgemeinbevölkerung. Diabetes mellitus ist mit einem erhöhten Risiko für kardiovaskuläre Erkrankungen und kardiovaskulären Tod verbunden, aber es ist nach wie vor unklar, welchen Anteil die Stoffwechselstörung allein an diesem erhöhten Risiko trägt und welcher auf Begleiterkrankungen wie Hypertonie zurückzuführen ist.
\end{abstract}

— Von den 10333 Patienten in der originalen Framingham-and-Offspring-Studie wurden jene herausgesucht, die älter als 35 Jahre waren und vor Studienbeginn keine kardiovaskulären Erkrankungen hatten.

Von 1145 Patienten mit Diabetes hatten 663 (58\%) bereits zum Zeitpunkt der Diagnosestellung eines Diabetes eine Hypertonie. Von 4154 Patienten starben in den Jahren der Nachbeobachtung 125 Patienten, 204 hatten ein kardiovaskuläres Ereignis. Diejenigen, die bereits zum Zeitpunkt der Diagnosestellung eines Diabetes an einer Hypertonie litten, zeigten eine signifikant höhere Rate an kardiovaskulärer Mortalität und kardiovaskulären Ereignissen im Vergleich zu normotensiven Diabetikern.

Nach Attestierung der demografischen und klinischen Kovarianten zeigten sich folgende Ergebnisse: Patienten mit Hypertonie und Diabetes hatten einen 72\%igen Risikoanstieg für die Gesamtmortalität und einen 57\%igen Risikoanstieg für kardiovaskuläre Ereignisse im Vergleich zu Diabetikern ohne Hypertonie. In einem mathematischen multivarianten Modell zeigte
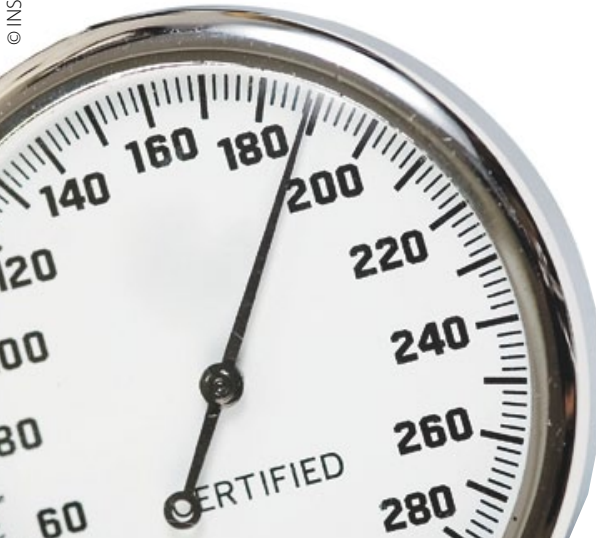

sich, dass Hypertonie und Diabetes jeweils unabhängige Risikofaktoren sind. Das sogenannte Population Attributable Risk war aber für Hypertonie bei Diabetes deutlich höher (30\% Gesamtmortalität und 25\% kardiovaskuläre Ereignisse) als bei Diabetes ohne Hypertonie (7\% Gesamtmortalität und 9\% kardiovaskuläre Ereignisse).

Diabetes mellitus ist zwar mit einem erhöhten Risiko für Tod und kardiovaskuläre Ereignisse assoziiert, das weitaus größere Risiko für diese Endpunkte ist jedoch einer gleichzeitig bestehenden arteriellen Hypertonie zuzuschreiben.

\section{Kommentar}

Die vorliegende Studie unterstreicht ein weiteres Mal die Notwendigkeit eines multifaktoriellen Therapienansatzes bei Patienten mit Diabetes mellitus Typ 2 und arterieller Hypertonie. Zweifellos stellt die Güte der diabetischen Stoffwechseleinstellung einen wesentlichen Grundpfeiler in der Prävention kardiovaskulärer Ereignisse dieser Patientengruppe dar. Im Fall einer gleichzeitig bestehenden arteriellen Hypertonie scheint jedoch die gute und zuverlässige Blutdruckeinstellung in dieser Hochrisikogruppe eine überragende Bedeutung einzunehmen. Bei allen in letzter Zeit geführten Diskussionen über neue Zielwerte und Zielkorridore bei Hypertonie und Diabetes sollte vor allem eines nicht vergessen werden: Die Notwendigkeit der Blutdrucksenkung per se bleibt bestehen und ist für diese Patientengruppe enorm wichtig.

W. KLEOPHAS =

\section{- G. Chen et al.}

Cardiovascular outcomes in framingham participants with diabetes: the importance of blood pressure. Hypertension 57 (2011) 891-897

Bluthochdruck ist für Diabetiker besonders gefährlich.

\section{ACHTUNG:}

\section{Hier muss der Dummy durch eine Anzeige ersetzt werden !!}

